BULLETIN OF THE

AMERICAN MATHEMATICAL SOCIETY

Volume 77, Number 3, May 1971

\title{
THE MANY-ONE EQUIVALENCE OF SOME GENERAL COMBINATORIAL DECISION PROBLEMS
}

\author{
BY C. E. HUGHES, ROSS OVERBEEK AND W. E. SINGLETARY ${ }^{1}$
}

Communicated by Dana Scott, September 28, 1970

1. Introduction. A decision problem for a combinatorial system shall denote a pair $(\phi, S)$ where $\phi$ is a specified kind of decision problem (e.g. derivability problem, halting problem, etc.) and $S$ is a combinatorial system. Two decision problems $\left(\phi_{1}, S_{1}\right),\left(\phi_{2}, S_{2}\right)$ are said to be of the same many-one degree (of unsolvability) if there exist effective many-one mappings $f$ and $g$ such that each instance of $\left(\phi_{1}, S_{1}\right)$ is reducible to an instance of $\left(\phi_{2}, S_{2}\right)$ via $f$ and each instance of $\left(\phi_{2}, S_{2}\right)$ is reducible to an instance of $\left(\phi_{1}, S_{1}\right)$ via $g$.

A general combinatorial decision problem, i.e., a decision problem for a class of combinatorial systems, shall denote a pair $(\phi, C)$ where $\phi$ is a specified kind of decision problem and $C$ is a class of combinatorial systems (e.g. Turing machines, semi-Thue systems, etc.). A general combinatorial decision problem $\left(\phi_{1}, C_{1}\right)$ is many-one reducible to another general combinatorial problem $\left(\phi_{2}, C_{2}\right)$ if there exists an effective one-one mapping $\psi$ of the problems $p$ associated with $\left(\phi_{1}, C_{1}\right)$ into the problems associated with $\left(\phi_{2}, C_{2}\right)$ such that $p$ is of the same many-one degree as $\psi(p) .\left(\phi_{1}, C_{1}\right)$ and $\left(\phi_{2}, C_{2}\right)$ are said to be many-one equivalent if each is many-one reducible to the other.

The reduction of one general combinatorial decision problem to another has been investigated by numerous authors. In particular, W. E. Singletary [15] has combined results of his own and those of others in such a way as to provide an effective proof of the (r.e.) equivalence of a number of general combinatorial decision problems. This former work has lead W. W. Boone to suggest that a stronger form of equivalence might exist between at least some subset of the problems considered. Our aim is to show that a number of these general problems are many-one equivalent. In addition, we indicate that these are, in a sense, best possible results.

AMS 1970 subject classifications. Primary 02F30, 02F43; Secondary 02F05, 02F15, $02 \mathrm{~F} 25,02 \mathrm{~F} 47$.

Key words and phrases. General combinatorial decision problem, many-one equivalence, many-one degrees, decision problem, halting problem, derivability problem, word problem, confluence problem, post correspondence problem, Turing machine, Markov algorithm, recursive function, semi-Thue system, Thue system.

${ }^{1}$ This research was partially supported by NSF Grant GP-23779. 
2. Preliminary definitions. If $R$ is a recursively enumerable set of nonnegative integers the decision problem for $R$ is the problem of determining for an arbitrary nonnegative integer $n$ whether or not $n$ is contained in $R$.

If $M$ is a Turing machine the derivability problem for $M$ is the problem of determining for arbitrary configurations $\alpha$ and $\beta$ of $M$ whether or not $M$, started in $\alpha$, will eventually reach $\beta$. The confluence problem for $M$ is the problem of determining for arbitrary configurations $\alpha$ and $\beta$ of $M$ whether or not there is a configuration $\gamma$ of $M$ such that $M$, started in $\alpha$, eventually reaches $\gamma$ and $M$, started in $\beta$, eventually reaches $\gamma$. The halting problem for $M$ is the problem of determining for an arbitrary configuration $\alpha$ of $M$ whether or not $M$, started in $\alpha$, eventually halts.

If $S$ is a Markov algorithm, semi-Thue system, tag system or Post normal system then the word problem for $S$ is the problem of determining for arbitrary $W_{1}$ and $W_{2}$ on the alphabet of $S$ whether or not $W_{2}$ is derivable from $W_{1}$ in $S$. For $S$ a Markov algorithm or semiThue system the confluence problem for $S$ is the problem of determining for arbitrary words $W_{1}$ and $W_{2}$ on the alphabet of $S$ whether or not there exists a word $W_{3}$ such that $W_{3}$ is derivable from both $W_{1}$ and $W_{2}$ in $S$. For $S$ a Markov algorithm or tag system, the halting problem for $S$ is the problem of determining for an arbitrary word $W$ whether or not $W$ is a mortal word of $S$.

A restricted Markov algorithm is a Markov algorithm in which no terminal productions are allowed. For further clarification see A. A. Markov [8].

If $S_{A}$ is a semi-Thue system, Thue system or Post normal system with axiom, the decision problem for $S_{A}$ is the problem of determining for an arbitrary word $W$ on the alphabet of $S_{A}$ whether or not $W$ is derivable from $A$ in $S_{A}$.

A correspondence class $C$ is an effective set of sequences of length $n$ (for some fixed $n$ ) of nonempty words over a finite alphabet. If $C$ is a correspondence class and $\alpha=\left(\alpha_{1}, \cdots, \alpha_{n}\right)$ and $\beta=\left(\beta_{1}, \cdots, \beta_{n}\right)$ are sequences of $C$, then there is a solution for $\alpha$ and $\beta$ if and only if there is a positive integer $k$ and a finite sequence $i_{1}, i_{2}, \cdots, i_{k}$ of integers $1,2, \cdots, n$ such that

$$
\alpha_{i_{1}} \alpha_{i_{2}} \cdots \alpha_{i_{k}}=\beta_{i_{1}} \beta_{i_{2}} \cdots \beta_{i_{k}} \text {. }
$$

The Post correspondence problem for a correspondence class $C$ is the problem of determining for arbitrary sequences $\alpha$ and $\beta$ of $C$ whether or not there is a solution for $\alpha$ and $\beta$. 
A correspondence class with axiom $C_{\alpha}$ is simply a correspondence class $C$ with a fixed sequence $\alpha$ of $C$ designated as axiom. The decision problem for a correspondence class with axiom $C_{\alpha}$ is the problem of determining for an arbitrary sequence $\beta$ of $C$ whether or not there is a solution for $\alpha$ and $\beta$.

3. Theorems and the outlines of the proofs. Let $R$ represent the general decision problem for recursively enumerable sets, $M_{D}$ the general derivability problem for Turing machines, $M_{H}, A_{H}$ and $P_{H}$ the general halting problems for Turing machines, restricted Markov algorithms and tag systems, respectively, $M_{C}$ and $A_{C}$ the general confluence problems for Turing machines and restricted Markov algorithms, respectively, $A_{W}, S_{W}, P_{W}$ and $N_{W}$ the general word problems for restricted Markov algorithms, semi-Thue systems, tag systems and Post normal systems, respectively, $C_{W}$ the general Post correspondence problem for correspondence classes, and $S_{A}, T_{A}, N_{A}$ and $C_{A}$ the general decision problems for semi-Thue systems with axiom, Thue systems with axiom, Post normal systems with axiom and correspondence classes with axiom, respectively.

THEOREM 1. Let $C$ be the class of Turing machines, Markov algorithms, semi-Thue systems or Post normal systems. Then it is not the case that every one-one degree of unsolvability is represented by the word (or derivability), halting or confluence problem of a system of $C$. Furthermore, it is not the case that every one-one degree is represented by the decision problem for either the class of semi-Thue systems with axiom or the class of Post normal systems with axiom.

IDEA OF PROOF. Let $S$ be the set of pairs of words $\left\{\left(W_{1}, W_{2}\right) W_{1}\right.$ $\left.\vdash_{M} W_{2}\right\}$ where $M$ is a system of one of the classes denoted by $C$. Then it is easy to show that the complement of $S$ contains an infinite r.e. set whenever the degree of $S$ is greater than 0 . Hence any one-one degree which contains a simple set is not represented by $S$. Analogous arguments may be used to establish the other results.

THEOREM 2. The general combinatorial decision problems $R, M_{D}$, $M_{H}, M_{C}, A_{W}, A_{H}, A_{C}, S_{W}, S_{C}, N_{W}, P_{W}, P_{H}, C_{W}, S_{A}, T_{A}, N_{A}$ and $C_{A}$ are many-one equivalent.

Corollary. For each triple $a_{1}, a_{2}, a_{8}$ of many-one degrees there exist

(i) a Turing machine whose halting, derivability and confluence problems are of degrees $a_{1}, a_{2}$, and $a_{3}$, respectively,

(ii) a Markov algorithm whose halting, word and confluence problems are of degrees $a_{1}, a_{2}$, and $a_{3}$, respectively, 
(iii) a semi-Thue system whose word and confluence problems are of degrees $a_{1}$ and $a_{2}$, respectively,

(iv) a tag system (and hence a Post normal system) whose halting and word problems are both of degree $a_{1}$,

(v) a Thue system (and hence a semi-Thue system) with axiom whose decision problem is of degree $a_{1}$, and

(vi) a Post normal system with axiom whose decision problem is of degree $a_{1}$.

Furthermore, this result is best possible in the sense that it does not hold for one-one degrees.

We shall indicate how to construct six sequences of reductions which may be linked together to obtain the results on many-one equivalence. These sequences may be represented diagrammatically as follows:

1. $R \stackrel{\mathrm{I}}{\longrightarrow} M_{D} \stackrel{\text { II }}{\longrightarrow} A_{W} \stackrel{\text { III }}{\longrightarrow} S_{W} \stackrel{\text { IV }}{\longrightarrow} C_{W} \stackrel{\text { V }}{\longrightarrow} R$

2. $R \stackrel{\mathrm{I}}{\longrightarrow} M_{H} \stackrel{\mathrm{VI}}{\longrightarrow} P_{W} \stackrel{\mathrm{VI}}{\longrightarrow} N_{W} \stackrel{\mathrm{IV}}{\longrightarrow} C_{W}$

3. $M_{H} \stackrel{\text { VII }}{\longrightarrow} T_{A} \stackrel{\text { VII }}{\longrightarrow} S_{A} \stackrel{\text { IV }}{\longrightarrow} N_{A} \stackrel{\text { IV }}{\longrightarrow} C_{A} \stackrel{\text { V }}{\longrightarrow} R$

4. $R \stackrel{\mathrm{I}}{\longrightarrow} M_{C} \stackrel{\text { II }}{\longrightarrow} A_{C} \stackrel{\text { III }}{\longrightarrow} S_{C} \stackrel{\text { VIII }}{\longrightarrow} R$

5. $M_{H} \stackrel{\text { II }}{\longrightarrow} A_{H} \stackrel{\text { VIII }}{\longrightarrow} R$

6. $M_{H} \stackrel{\text { VI }}{\longrightarrow} P_{H} \stackrel{\text { VIII }}{\longrightarrow} R$

where each arrow represents an effective mapping which when applied to any problem associated with the general combinatorial decision problem at the tail of the arrow will produce a problem of the same many-one degree associated with the general combinatorial decision problem represented at the head of the arrow. The numbers above the arrows indicate the order in which these reductions will be given.

I. The idea is to construct, for each unary recursive function $f$, a Turing machine $M$, which strongly computes $f$. From $M$, three different machines $M_{1}, M_{2}$ and $M_{3}$ are constructed such that the decision problem for the range of $f$, the derivability problem for $M_{1}$, the halting problem for $M_{2}$ and the confluence problem for $M_{3}$ are each of the same many-one degree. In addition these machines are such that the problems not being considered in each one are all solvable. Finally, for any three total recursive functions $f_{1}, f_{2}$ and $f_{3}$, one may combine three machines, constructed as above, to obtain a machine whose 
halting, derivability and confluence problems are of the same manyone degrees as the decision problems for the range of $f_{1}, f_{2}$ and $f_{3}$, respectively. This is essentially a refinement of Shepherdson's work [14].

II. The idea is to encode configurations of the Turing machine onto a recursive subset, called "normal words," of the words of the restricted Markov algorithm. Using the deterministic nature of Markov algorithms, special symbols act as shuttles which both carry out the simulation on normal words and fix up "garbage words."

III. This reduction is performed in two steps. A semi-Thue system is constructed which, in the manner of Shepherdson [14], uses left, right, beginning and end symbols to simulate the restricted Markov algorithm in such a way as to preserve unbounded truth table degrees for the word and confluence problems. Productions are then added which allow the leftmost end symbol to arbitrarily destroy or create any symbols on its right. This then allows us to consider only words with one segment and hence to preserve many-one degrees.

IV. These reductions are realized by modifications of those carried out by Cudia and Singletary [5]. The crucial step is in noting that, whenever we arrive at the correspondence problem, many-one reducibility may be maintained by choosing an appropriate recursive class of sequences.

V. Cudia and Singletary [4] have shown that the general Post correspondence problem for correspondence classes is many-one reducible to the general definition problem for partial recursive functions. A minor modification of this technique will prove the desired result for the general decision problem for correspondence classes with axiom.

VI. Aanderaa and Belsnes [1] have announced that the halting problem for tag systems may have an arbitrary r.e. degree of unsolvability. Expanding on their method a tag system may be constructed to simulate a Turing machine in such a manner that its word problem is of the same many-one degree as its halting problem and its halting problem is of the same many-one degree as that of the Turing machine.

VII. For an arbitrary Turing machine $M$, a semi-Thue system $S$ is generated such that, for any arbitrary configuration $\alpha$ of $M$, $h \alpha h \vdash_{s} h q_{H} h$ if and only if $\alpha$ is a mortal configuration of $M . S$ is monogenic and has the property that if we let $\bar{S}$ be the semi-Thue system which has the inverse of every production of $S$ then $h q_{H} h \vdash \bar{s} h \alpha h$, for $\alpha$ a configuration of $M$, if and only if $\alpha$ is mortal. A Thue system $T$ with axiom $h q_{H} h$ is then defined which has exactly 
the productions of $S$ and $\bar{S}$. Using an argument of Post [13] we have that the words derivable from the axiom of $T$ are exactly those derivable from this axiom in $\bar{S}$. This gives us the desired result.

VIII. In the indicated places it is clear that it is a straightforward exercise to write partial recursive functions whose definition problems are of the same many-one degrees as the appropriate problems.

\section{BIBLIOGRAPHY}

1. D. Belsnes and S. O. Aanderaa, Decision problems for tag systems, Notices Amer. Math. Soc. 14 (1967), 950. Abstract \#67-698.

2. P. Axt and W. E. Singletary, On deterministic normal systems, Z. Math. Logik Grundlagen Math. 15 (1969), 49-62.

3. W. W. Boone, Word problems and recursively enumerable degrees of unsolvability. A first paper on Thue systems, Ann. of Math. (2) 83 (1966), 520-571; Bull. Amer. Math. Soc. 68 (1962), 616-623. MR 34 \#1381.

4. D. F. Cudia and W. E. Singletary, The Post correspondence problem, J. Symbolic Logic 33 (1968), 418-430. MR 39 \#5367.

5. - Degrees of unsolvability in formal grammars, J. Assoc. Comput. Mach. 15 (1968), 680-692. MR 38 \#5534.

6. M. Davis, Computability and unsolvability, McGraw-Hill Series in Information Processing and Computers, McGraw-Hill, New York, 1958. MR 23 \#A1525.

7. P. K. Hooper, Monogenic Post normal systems of arbitrary degree, J. Assoc. Comput. Mach. 13 (1966), 359-363. MR 33 \#3846.

8. A. A. Markov, Theory of algorithms, Trudy Mat. Inst. Steklov. 42 (1954); English transl., Israel Program Scientific Translations, Jerusalem, 1961. MR 17, 1038; MR 24 \#A2527.

9. S. Ju. Maslov, On E. L. Post's "tag problem," Trudy Mat. Inst. Steklov, 72 (1964), 57-68; English transl., Amer. Math. Soc. Transl. (2) 97 (1970), 1-14. MR 34 $\# 4129$.

10. M. L. Minsky, Recursive unsolvability of Post's problem of "tag" and other topics in theory of Turing machines, Ann. of Math. (2) 74 (1961), 437-455. MR 25 \#3825.

11. E. L. Post, Formal reductions of the general combinatorial decision problem, Amer. J. Math. 65 (1943), 197-215. MR 4, 209.

12. - A variant of a recursively unsolvable problem, Bull. Amer. Math. Soc. 52 (1946), 264-268, MR 7, 405.

13. - Recursive unsolvability of a problem of Thue, J. Symbolic Logic 12 (1947), 1-11. MR 8, 558.

14. J. C. Shepherdson, Machine configuration and word problems of given degree of unsolvability, Z. Math. Logik Grundlagen Math. 11 (1965), 149-175. MR 30 \#4681.

15. W. E. Singletary, The equivalence of some general combinatorial decision problems, Bull. Amer. Math. Soc. 73 (1967), 446-451. MR 35 \#1477.

16. A. Yasuhara, $A$ remark on Post normal systems, J. Assoc. Comput. Mach. 1 (1967), 167-171. MR 35 \#4104.

Pennsylvania State University, University Park, Pennsylvania 16802 